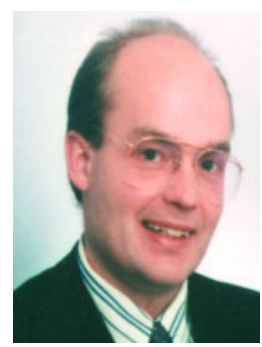

B. Kardels

Kasuistik

\title{
Notarzteinsatz bei einer Frau mit Stiff-Man-Syndrom
}

B. Kardels, K.-H. Beine

St. Marien-Hospital Hamm gGmbH, Klinik für Psychiatrie und Psychotherapie, Universität Witten-Herdecke

NOTFALL \& HAUSARZTMEDIZIN 2004; 30: B 437-B 439

Geschildert wird ein Notarzteinsatz bei einer 33-jährigen Patientin, die an der Autoimmunerkrankung Stiff-Man-Syndrom und einer Anpassungsstörung litt. Sie berichtete über Myoklonien in den Armen, Beinen und im Rücken. Sie stellte dem Notarzt die dafür nötigen Medikamente zur Verfügung, sodass eine adäquate medikamentöse Therapie eingeleitet werden konnte. Die Patientin war dem Notarzt aus früheren Klinikaufenthalten bekannt. Das StiffMan-Syndrom ist häufig mit einer psychiatrischen Erkrankung vergesellschaftet. Liegt eine neurologische und psychiatrische Erkrankung vor, so sollte der Transport in ein Krankenhaus mit beiden Abteilungen erfolgen.

Suizidversuch erfolgt. Die Patientin hatte sich drei Jahre zuvor in suizidaler Absicht Schnittverletzungen am linken Handgelenk zugezogen und mehrere Mestinon ${ }^{\circledR}$-Tabletten eingenommen. Damals war eine Zwangseinweisung erfolgt.

Bei der körperlichen Untersuchung war die Extremitätenbewegung an beiden Armen und Beinen stark vermindert und es bestand eine fluktuierende Muskelsteifigkeit mit reflektorischen Spasmen bei Berührung. Die Patientin wies beidseitig positive Babinski-Zeichen auf. Die Pupillen waren weit und die Augenmotilität eingeschränkt. Der Ruheblutdruck betrug 130/80 mmHg bei einer Herzfrequenz von 90 Schlägen pro Minute. Die periphere Sauerstoffsättigung am Finger lag bei 95\%.
Das abgeleitete EKG zeigte einen Sinusrhythmus an. Die Atmung war unauffällig und es ergaben sich keine Hinweise auf äußere Verletzungen.

Der anwesende Ehemann berichtete, dass seine Frau einen Port implantiert bekommen habe, da sie schon öfters „unter solchen Schmerzattacken" gelitten habe. Der behandelnde Hausarzt habe deshalb auch Medikamente verschrieben und hinterlegt. Der Ehemann öffnete einen roten Kulturbeutel mit 3 Morphin ${ }^{\circledR}$ Ampullen mit je 10 mg, 5 Rivotril ${ }^{\circledR}$ Ampullen mit je $1 \mathrm{mg}$ und 3 Psyquil $^{\circledR}$-Ampullen zu je $10 \mathrm{mg}$. Die Patientin forderte den Notarzt auf, einen Teil der Medikamente zu spritzen. Dies sei beim letzten Anfall auch durch den anwesenden Arzt erfolgt und es habe ihr geholfen. 
Der Notarzt punktierte zunächst den angelegten Port und infundierte ihr $500 \mathrm{ml}$ Ringer-Laktat ${ }^{\circledR}$-Lösung. Anschließend verabreichte er ihr 10 mg Morphin ${ }^{\circledR}$ und 4 mg Rivotril ${ }^{\circledR}$ fraktioniert. Zusätzlich erhielt die Patientin 4 Liter Sauerstoff über eine angelegte Nasensonde. Um 17.08 Uhr erfolgte der Transport per RTW mit Arztbegleitung in die neurologische Abteilung des nächstgelegenen Krankenhauses unter den Diagnosen Stiff-Man-Syndrom und Anpassungsstörung. Um 17.20 Uhr wurde die Patientin vom Notarzt an den Aufnahmearzt im Krankenhaus mit einer kurzen Schilderung des Einsatzablaufes übergeben.

\section{Therapie und Verlauf}

In der Klinik berichtete die Kranke über zunehmende Crampi der Rückenmuskulatur und einen aufgetretenen Kontrollverlust der Beine mit Unfähigkeit des freien Sitzens. Bei der körperlichen Untersuchung war der Allgemeinzustand reduziert, die Wangen gerötet, das vesikuläre Atemgeräusch basal über beiden Lungenflügeln abgeschwächt und die Wirbelsäule im LWS-Bereich klopfschmerzhaft.

Die neurologische Untersuchung ergab folgende Pathologika: Eingeschränkte Augenmotilität beidseits mit mydriatischen Pupillen sowie eine Hypomimie. Die Muskeleigenreflexe an den Armen und Beinen waren nicht sicher auslösbar. Es bestand eine Tetraparese im Bereich der Arme mit Kraftgrad 3-4 und im Bereich der Beine mit Kraftgrad 1-2. Arm- und Beinhalteversuche waren nicht durchführbar. Der Muskeltonus war rechtsbetont im Bereich der Beine erhöht. An den distalen Extremitätenabschnitten wurde eine Hypästhesie und eine Hypalgesie angegeben. Zum Teil wies die Patientin eine intermittierende dystone Hand- und Fingerstellung auf.

In der Aufnahmesituation war die Patientin zu Ort, Zeit und Person orientiert. Der formale und inhaltliche Gedankengang war intakt. Der Antrieb war reduziert. Die Stimmungslage war zum Depressiven verschoben. Eine akute Suizidalität war nicht sicher ausschließbar.
Die durchgeführten Untersuchungen während des Klinikaufenthaltes mittels Elektroneurographie ergaben eine geringe sensible Polyneuropathie des rechten Nervus radialis und Nervus medianus. Die Elektromyographie des Musculus biceps brachii rechts ergab Muskelantwortpotenziale von normaler Dauer, Amplitude und Polyphasie. Die veranlasste MRT-Untersuchung der LWS wies bis auf Schmorlsche Knorpelknötchen im thorakalen Übergang, wie sie für einen Morbus Scheuermann typisch sind, keine Pathologika auf.

Therapeutisch erfolgte eine parenterale Infusionstherapie mit Rivotril $^{\circledR}$ und Dipidolor ${ }^{\circledR}$. Zusätzlich wurde eine intrathekale Baclofenpumpe implantiert und es erfolgte eine Gabe von $500 \mu \mathrm{g}$ Baclofen ${ }^{\circledR}$ innerhalb von 24 Stunden. Des Weiteren erhielt die Kranke Mestinon retard ${ }^{\circledR}$ in einer Dosierung von 3-mal $90 \mathrm{mg}$ pro Tag. Nach sechs Tagen wurde die Patientin in die ambulante Weiterbehandlung eines niedergelassenen Nervenarztes entlassen.

\section{Diskussion}

Das Stiff-Man-Syndrom ist eine seltene und schwere Erkrankung, die erstmals von Moersch und Woltman phänomenologisch beschrieben wurde $(1,2,3)$. Bereits im Jahre 1966 wurde von Young die Möglichkeit einer autoimmunen Genese des Stiff-Man-Syndroms diskutiert (1). Heute wird für diese Erkrankung in der Literatur eine autoimmune Genese angenommen $(1,3,4)$. In $60-$ $80 \%$ der Fälle werden Antikörper gegen die Glutamatdecarboxylase, welche die Umwandlung von Glutamat in Gammaaminobuttersäure katalysiert, gefunden $(4,6,7,8)$. Die Erkrankung kann mit einem Diabetes mellitus, einem Mamma- oder Lungenkarzinom assoziiert sein $(1,3,5$, 9). Das Manifestationsalter wird in der Literatur mit 14-72 Jahren angegeben und die Erkrankungsdauer zwischen einem und 36 Jahren (6). Andere Autoren legen das Durchschnittsalter auf 41,2 Jahre fest und gehen von einem durchschnittlichen Zeitraum von 6,2 Jahren bis zur Diagnosenstellung aus (7). Unsere Patientin war zum Zeitpunkt des
Notarzteinsatzes 33 Jahre alt, wobei die Diagnose bereits drei Jahre früher gestellt worden war.

Literaturberichten zur Folge ist das weibliche Geschlecht häufiger vom Stiff-Man-Syndrom befallen (6, 7). Klinisch ist die Krankheit durch fluktuierende muskuläre Rigidität sowie durch spontane und reflektorische Spasmen gekennzeichnet (6). Einschießende Spasmen können zu Subluxationen, Ankylosen, Verbiegungen der Wirbelsäule und Frakturen führen (6,7). Die Assoziation mit neurologischen Symptomen wie Augenmotilitätsstörungen, Paresen, Ataxien, ein- oder beidseitigen Babinski-Zehenzeichen und Sensibilitätsstörungen können vorkommen (6). In unserem Patientenbeispiel konnte der Notarzt am Einsatzort die obligatorischen spontanen Spasmen und die Muskelsteifigkeit feststellen. Zusätzlich wies die Kranke positive Babinski-Zeichen beidseitig auf. Auch zum Aufnahmezeitpunkt in der neurologischen Zielklinik war die initiale Augenmotilitätsstörung weiterhin feststellbar.

Die Assoziation mit neurologischen Symptomen wird heute als „Plus"-Variante des Stiff-Man-Syndroms verstanden und als progressive Enzephalomyelitis mit Rigidität und Myoklonien bezeichnet (3, 6, 10). Korrespondierend wird die Muskelsteifigkeit nur einer Gliedmaße als Stiff-Limb-Syndrom bezeichnet und als „Minus”-Variante aufgefasst.

Das Stiff-Man-Syndrom kann mit einer vegetativen Entgleisung einhergehen $(6,11)$. Sympathogen bedingt können eine Mydriasis, eine ausgeprägte Hyperhidrosis, Tachykardien, eine Tachypnoe, arterielle Hypertensionen, Muskelspasmen sowie ein akuter Kreislaufschock auftreten (6). In einer Studie von Meinck verstarben immerhin 10\% der Patienten an einer akuten autonomen Entgleisung (6). In unserem Patientenbeispiel war die Patientin nicht vegetativ entgleist.

Häufig ist das Stiff-Man-Syndrom zusätzlich mit einer psychiatrischen Erkrankungen assoziiert. In der Literatur wurden Angsterkrankungen mit Agoraphobie, Depressionen und Substanzmissbrauch als Be- 
gleiterkrankung beschrieben $(12,13$, 14). Bei unserer Patientin war eine Anpassungsstörung aus einem früheren psychiatrischen Klinikaufenthalt bekannt. Bei den Anpassungsstörungen handelt es sich definitionsgemäß um Zustände von subjektivem Leiden und emotionaler Beeinträchtigung, die soziale Funktionen und Leistungen behindern und während des Anpassungsprozesses nach einer entscheidenden Lebensveränderung oder nach belastenden Lebensereignissen - auch schwerer körperlichen Erkrankung auftreten (15). Symptome sind depressive Stimmung, Angst, Besorgnis, Sorge, mit der gegenwärtigen Situation nicht zurechtzukommen, eventuell auch Probleme bei der Bewältigung der alltäglichen Routine (15). Sicherlich wies unsere Patientin einen Substanzmissbrauch von Rivotril und Morphin auf, sonst hätte sie diese Medikamente nicht in so hohen Dosen in ihrem Kulturbeutel aufbewahrt. Da Anpassungsstörungen mit depressiven Episoden und Suizidalität vergesellschaftet sein können, wäre ein vollendeter Suizid in unserem Patientenbeispiel durchaus denkbar gewesen.

\section{- Fazit}

Notärzte werden im Rettungsdienst mit den verschiedensten Krankheitsbildern konfrontiert. Bei unserer Kasuistik traf der Notarzt auf eine Patientin mit einem StiffMan-Syndrom und einer Anpassungsstörung. Präklinisch verabreichte er ihr Opiate, Tranquillizer und Neuroleptika, was durchaus auch indiziert war. Dennoch sollte er bei der Auswahl des Zielkrankenhauses darauf achten, dass der Patient in ein Krankenhaus mit den Disziplinen Neurologie und Psychiatrie transportiert wird. Somit kann das Stiff-Man-Syndrom in der Neurologie - je nach Symptomatik - mit Tranquillizern, Gabapentin, Baclophen, Kortison, Imunglobulinen oder Plasmaphorese behandelt werden $(1,2,4,6,7)$ und in der Psychiatrie kann aufgrund der Anpassungsstörung eine adäquate Psychotherapie und gegebenenfalls eine psychopharmakologische Medikamentengabe erfolgen (15).

\section{Summary}

Reported is of an emergency case of a 33 years old female, who was suffering of an autoimmune disorder of a stiff-man syndrome and an adjustment disorder. She was reporting of myocloni in the arms, legs and the back. She was giving the right medications to the emergency medical doctor, so that she could be treated adequately. The emergency medical doctor has seen this patient before because of former hospitalizations. The stiff-man syndrome is often associated with a psychiatric disorder. Is there a neurological and a psychiatric disorder existing, then the patient should be transported in a hospital with both departments.

\section{Literatur}

1. Seissler J, Scherbaum WA. Das StiffMan-Syndrom aus internistischer und immunologischer Sicht. Der Internist 2000; 41: 460-466

2. Armon C, Swanson JW, McLean JM et al. Subacute encephalomyelitis presenting as stiff-person syndrome: Clinical, polygraphic, and pathologic correlations. Movement Disorders 1996; 11: 701-709

3. Folli F. Stiff man syndrome, 40 years later. Journal of Neurology, Neurosurgery, and Psychiatry 1998; 65: 618

4. Diedrich U. Rhythmische Kreislaufdysregulation bei Stiff-man-Syndrom. Nervenarzt 1996; 67: 1027-1029

5. Folli F, Solimena M, Cofiell R et al. Autoantibodies to a 128-kd synaptic protein in three women with the stiff-man syndrome and breast cancer. The New England Journal of Medicine 1993; 328: 546-551

6. Meinck HM. Das Stiff-Man-Syndrom aus neurologischer Sicht. Der Internist 2000; 41: 455-459

7. Dalakas MC, Fujii M, Li M, McElroy B. The clinical spectrum of anti-GAD antibody-positive patients with stiff-person syndrome. Neurology 2000; 55: 1531-1535

8. Silverman IE. Paraneoplastic stiff limb syndrome. Journal of Neurology, Neurosurgery and Psychiatry 1999; 67: 126-127

9. Saiz A, Dalmau J, Butler MH et al. Antiamphiphysin I antibodies in patients with paraneoplastic neurological disorders associated with small cell lung carcinoma. Journal of Neurology, Neurosurgery and Psychiatry 1999; 66: 214-217

10. Barker RA, Revesz T, Thom M et al. Review of 23 patients affected by the stiff man syndrome: clinical subdivision into stiff trunk (man) syndrome, stiff limb syndrome, and progressive encephalomyelitis with rigidity. Journal of Neurology, Neurosurgery and Psychiatry 1998; 65: 633-640

12. Göppert D, Gardill K, Beischer W, Wiethölter H. Stiff-man-Syndrom mit Diabetes mellitus Typ 1 und Autoimmunthyreoditis.
Deutsche Medizinische Wochenschrift 2000; 125: 826-829

13. Tinsley JA, Barth EM, Black JL, Williams $D E$. Psychiatric consultations in stiff-man syndrome. Journal of Clinical Psychiatry 1997; 58: 444-449

14. Henningsen $P$, Meinck HM. Specific phobia is a frequent non-motor feature in stiff man syndrome. Journal of Neurology, Neurosurgery and Psychiatry 2003; 74: 462-465

14. Gerschlager W, Schrag A, Brown P. Quality of life in stiff-person syndrome. Movement Disorders 2002; 17: 1064-1067

15. Dilling H, Reimer C, Arolt V. Basiswissen Psychiatrie und Psychotherapie. 4. Auflage. Berlin, Heidelberg, New York, Barcelona, Hongkong, London, Mailand, Paris, Singapur, Tokio: Springer Verlag, 2001

\section{Anschrift für die Verfasser}

Dr. med. Björn Kardels,

Prof. Dr. med. Karl-Heinz Beine

St. Marien-Hospital Hamm gGmbH

Klinik für Psychiatrie und Psychotherapie,

Universität Witten-Herdecke

Knappenstraße 19

59071 Hamm

E-mail: Bjoern.Kardels@marienhospitalhamm.de

Diese Kasuistik erschien zuerst in: Der Notarzt 2004; 20: 100-102.

Der Nachdruck erfolgt mit freundlicher Genehmigung der Autoren. 